\title{
Stability Analysis of a Rigid Body with a Flexible Attachment Using the Energy-Casimir Method
}

\author{
Thomas A. Posbergh" and P.S. Krishnaprasad* \\ Jerrold E. Marsden ${ }^{\dagger}$
}

\begin{abstract}
${ }^{1}$
We consider a system consisting of a rigid body to which a linear extensible shear beam is attached. For such a system the Energy-Casimir method can be used to investigate the stability of the equilibria. In the case we consider, it can be shown that a test for (formal) stability reduces to checking the positive definiteness of two matrices which depend on the parameters of the system and the particular equilibrium about which the stability is to be ascertained.
\end{abstract}

\section{Introduction}

We consider a rigid body to which a long, flexible appendage is attached. A coordinate reference frame is fixed in the rigid body with the origin at the center of mass of the rigid body. The flexible attachment is assumed to lie along the second coordinate axis when the configuration is at rest. (see Figure 1.) The equations of motion for such a configuration, under suitable assumptions and with the appendage modeled as a linear extensible shear beam, are derived by Krishnaprasad and Marsden in [2]. In deriving the equations of motion they use Hamiltonian methods in the context of Poisson manifolds and reduction. (see [2] for the explicit formula for the Poisson brackets involved.) If we assume that the momentum of the system which arises from the appendage rotating with the

\footnotetext{
- Research supported in part by National Science Foundation grant OIR-85-00108 to the Systems Research Center, AFOSR-URI grant AFOSR-87-0073, and by a Deaign Project Grant from NASA Headquarters through the Universities Space Regearch Association.

${ }^{t_{R}}$ esearch supported in part by DOE Contract DE-AT03-85ER-12097 and by AFOSR-URI grant AFOSR-87-0073

'This paper is in final form and no version of it is submitted elsewhere.
}

(C) 1987 American Mathematical Society $0271-4132 / 87 \$ 1.00+\$ .25$ per page 


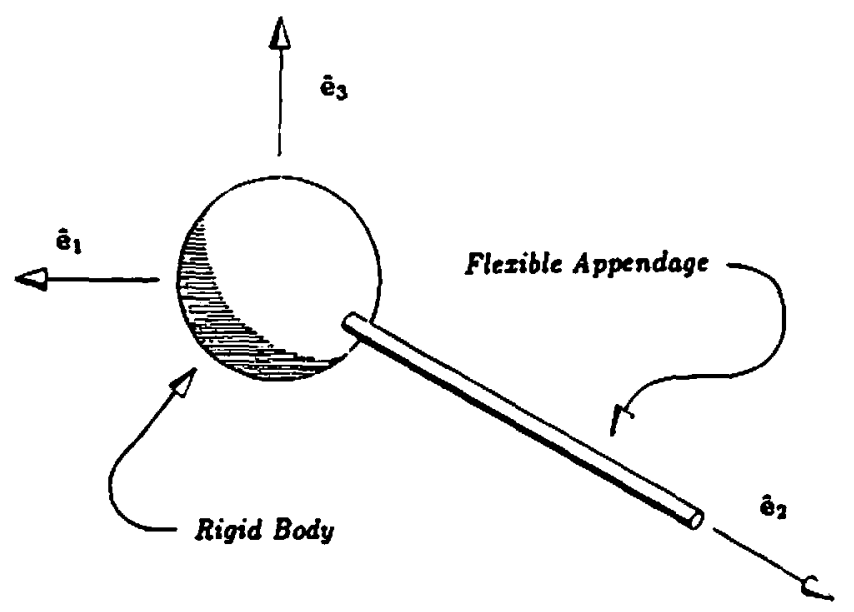

Figure 1: The Geometry of the Configuration

rigid body is negligible, then our Hamiltonian is of the form

$$
H=\frac{1}{2} J^{-1} p \cdot p+\frac{1}{2} \int_{0}^{e} \frac{\|m(s)\|^{2}}{\rho_{0}} d s+\frac{1}{2} \int_{0}^{e} \mathbf{K} \frac{\partial \mathbf{r}}{\partial s} \cdot \frac{\partial r}{\partial s} d s .
$$

We assume that $J$ is the inertia matrix of the rigid body and that $\rho_{0}$ is the uniform mass per unit length of the attached appendage of length $\ell$. The reduced phase space is coordinated at any time by $w$, the convected angular velocity vector of the rigid body; $r(s)$, the convected displacement of the shear beam at a point $s, 0 \leq s \leq \ell_{i}$ and $m(s)$ the momentum density of shear beam at the point s. The vector $p$ is the body angular momentum vector of the rigid body, thus $\mathbf{p}=\mathbf{J} \boldsymbol{\omega}$. Finally, $\mathbf{K}$ is the diagonal matrix of elastic coefficients.

In our investigation we are interested in the stability of the system about equilibria points. These equilibria will satisfy,

$$
\begin{aligned}
0=J \omega \times \omega+\mathbf{J} \times\left.\mathbf{K} \frac{\partial r}{\partial s}\right|_{\bullet}=0-r(l) \times \mathbf{K} \hat{e}_{2}+\int_{0}^{l} \frac{\partial r}{\partial s} \times \mathbf{K} \frac{\partial r}{\partial s} d s \\
0=\frac{1}{\rho_{0}} \mathbf{m}+\mathbf{r} \times \omega \\
0=\mathbf{K} \frac{\partial^{2} r}{\partial s^{2}}+\mathbf{m} \times \omega .
\end{aligned}
$$


Two boundary values are associated with these equations,

$$
\left.\frac{\partial r}{\partial s}\right|_{\rho=\ell}=\left[\begin{array}{l}
0 \\
1 \\
0
\end{array}\right]=e_{2}, \text { and }\left.\right|_{a=0}=\left[\begin{array}{l}
0 \\
a \\
0
\end{array}\right]=a \text {. }
$$

In $\{2\}$, a stability algorithm based on the Energy-Casimir method was applied to a specific family of equilibria (see subsection 4.2 below). The essence of the stability algorithm is to recognize that the relevant Poisson structure $\{\cdot, \cdot\}$ admits nontrivial Casimirs i.e. functions $F$ that Poisson-commute with any function of the phase space. It follows that these are also conserved quantities for the dynamics of (1). Specific Casimirs $C_{\phi}$ may be found such that the relative equilibria defined by (2)-(4) are critical pointa of $\left(H+C_{\phi}\right)$ on the reduced phase space. Formal stability follows from establishing definiteness conditions for the second variation $D^{2}\left(H+C_{\phi}\right)$ at the relative equilibria. To establish rigorous nonlinear stability, one has to carry out certain convexity estimates as in [2].

The purpose of this paper is to establish a systematic procedure for carrying out the formal stability step for arbitrary equilibria satisfying the equations (2)(4). This has useful applications in the engineering context where the model at hand represents the mechanics of a spinning spacecraft with a flexible attachment (such as a boom for carrying instruments or an antenna). See [2]. for related remarks and references. The procedure derived here recovers the results of [2] when applied to the specific example considered there. (see subsection 4.2 below.)

\section{Computation of the First and Second Variations}

In this section we compute the first and second variations of the Hamiltonian plus the Casimir function, $H+C_{\phi}$. From the previous definitions of these we know

$$
H=\frac{1}{2} \mathrm{~J}^{-1} \mathbf{p} \cdot+\frac{1}{2} \int_{0}^{l} \frac{\|\mathrm{m}(s)\|^{2}}{\rho_{0}} d s+\frac{1}{2} \int_{0}^{l} \mathbf{K} \frac{\partial \mathrm{r}}{\partial s} \cdot \frac{\partial \mathbf{r}}{\partial s} d s,
$$

and the Casimir function may be taken to be

$$
C_{\phi}=\frac{1}{2} \phi\left(\left\|p+\int_{0}^{l} \mathbf{r} \times \mathbf{m} d s\right\|^{2}\right)
$$

We will denote the first and second variations by $D\left(H+\mathrm{C}_{\phi}\right)$, and $D^{2}\left(H+C_{\phi}\right)$. Note that because of the distributed nature of the system we are dealing with we will need to compute variational derivatives instead of ordinary gradients. 


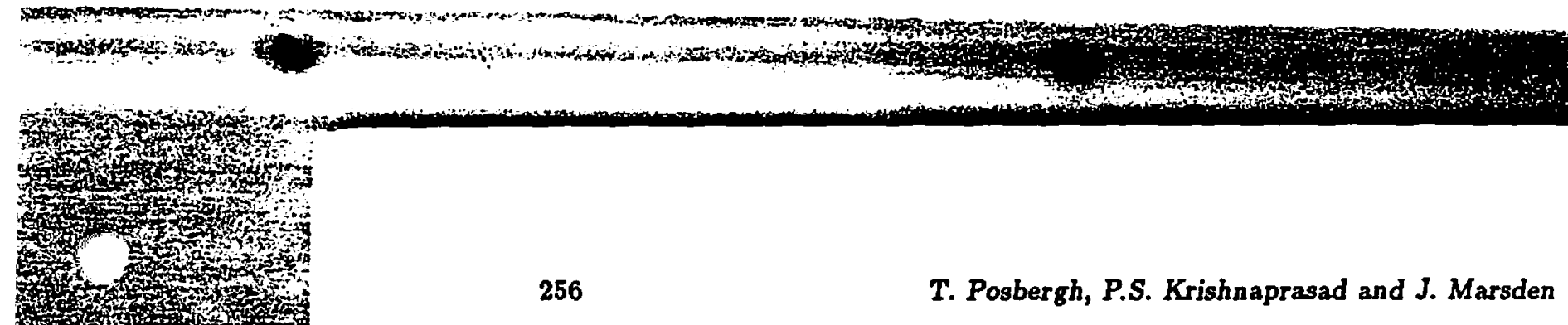

\subsection{Computation of the First Variation}

For the integrals in the Hamiltonian we consider variational differentials by

$$
D f(x)=\lim _{e \rightarrow 0} \frac{f(x+\epsilon h)-f(x)}{\epsilon}=\int_{0}^{\ell} \frac{\delta f}{\delta x} \cdot \delta x d s .
$$

Thus, letting

$$
f_{2}=\frac{1}{2} \int_{0}^{e} \frac{\|\mathbf{m}(s)\|^{2}}{\rho_{0}} d s,
$$

then

$$
D f_{2}(m)=\int_{0}^{l} \frac{1}{\rho_{0}} m \cdot \delta m d s
$$

Similarly, let

$$
\begin{aligned}
f_{3}(\mathbf{r}) & =\frac{1}{2} \int_{0}^{e} \mathbf{K} \frac{\partial \mathbf{x}}{\partial s} \cdot \frac{\partial \mathbf{r}}{\partial s} d s \\
D f_{3}(r) & =\int_{0}^{e} \mathbf{K} \frac{\partial \mathbf{r}}{\partial s} \cdot \frac{\partial \delta \mathbf{r}}{\partial s} d s .
\end{aligned}
$$

If we integrate this equation by parts with the boundary conditions $\delta r(\ell)=$ $\delta r(0)=0$, we get

$$
D f_{3}(\mathbf{r})=-\int_{0}^{e} \mathbf{K} \frac{\partial^{2} \mathbf{r}}{\partial s^{2}} \cdot \delta \mathbf{r} d s
$$

For the integral term in the Casimir function we are taking variational derivatives of a cross product term. If we define

$$
\|\alpha\|^{2}=\left\|p+\int_{0}^{l} \mathbf{r} \times \mathbf{m} d s\right\|^{2},
$$

then

$$
D\|\alpha\|^{2}=2 \alpha \cdot\left(\delta \mathrm{p}+\int_{0}^{l} \mathbf{r} \times \delta \mathrm{m} d s+\int_{0}^{l} \delta \mathrm{r} \times \mathbf{m} d s\right) .
$$

If we combine all of the above we get the expression for the first variation

$$
\begin{aligned}
D(H & \left.+C_{\phi}\right)=\mathbf{J}^{-1} \mathbf{p} \cdot \delta \mathrm{p}+\int_{0}^{l} \frac{1}{\rho_{0}} \mathbf{m} \cdot \delta \mathbf{m} d s-\int_{0}^{l} \mathbf{K} \frac{\partial^{2} s}{\partial s^{2}} \cdot \delta \mathbf{r} d s \\
& +\phi^{\prime}\left(\|\boldsymbol{\alpha}\|^{2}\right) \alpha \cdot\left(\delta \mathbf{p}+\int_{0}^{l} \mathbf{r} \times \delta \mathbf{m} d s+\int_{0}^{l} \delta \mathbf{r} \times \mathbf{m} d s\right) .
\end{aligned}
$$




\subsection{Computation of the Second Variation}

For the second variation, the starting point is the expression for the first variation. The terms arising from the original Hamiltonian are straight forward to compute, they are

$$
\begin{aligned}
D\left(\mathrm{~J}^{-1} \mathrm{p} \cdot \delta \mathrm{p}\right) & =\mathrm{J}^{-1} \delta \mathrm{p} \cdot \delta \mathrm{p}, \\
D\left(\int_{0}^{\ell} \frac{1}{\rho_{0}} \mathrm{~m} \cdot \delta \mathrm{m} d s\right) & =\int_{0}^{\ell} \frac{1}{\rho_{0}} \delta \mathrm{m} \cdot \delta \mathrm{m} d s, \\
D\left(\int_{0}^{l} \mathrm{~K} \frac{\partial^{2} r}{\partial s^{2}} \cdot \delta \mathrm{r} d s\right) & =\int_{0}^{\ell} \mathbf{K} \frac{\partial^{2} \delta \mathrm{r}}{\partial s^{2}} \cdot \delta \mathrm{r} d s .
\end{aligned}
$$

Note that we can use the boundary conditions on $\delta \mathbf{r}$ to get

$$
\int_{0}^{\ell} K \frac{\partial^{2} \delta \mathbf{r}}{\partial s^{2}} \cdot \delta r d s=-\int_{0}^{l} K \frac{\partial \delta r}{\partial s} \cdot \frac{\partial \delta r}{\partial s} d s .
$$

Next we consider the component which arises from the Casimir function which we added to the Hamiltonian. From the first factor of this term we compute,

$$
D \phi^{\prime}(\alpha)=2 \phi^{\prime \prime}\left(\|\alpha\|^{2}\right) \alpha \cdot\left(\delta \mathrm{p}+\int_{0}^{l} \mathrm{r} \times \delta \mathrm{m} d s+\int_{0}^{l} \delta \mathrm{r} \times \mathrm{m} d s .\right)
$$

From the second factor of the Casimir term we compute

$$
\begin{gathered}
D\left(\alpha \cdot\left(\delta \mathrm{p}+\int_{0}^{e} \mathrm{r} \times \delta \mathrm{m} d s+\int_{0}^{e} \delta \mathrm{r} \times \mathrm{m} d s\right)\right)= \\
\left\|\delta \mathrm{p}+\int_{0}^{e} \mathrm{r} \times \delta \mathrm{m} d s+\int_{0}^{e} \delta \mathrm{r} \times \mathrm{m} d s\right\|^{2} \\
+2\left(\mathrm{p}+\int_{0}^{e} \mathrm{r} \times \mathrm{m} d s\right) \cdot\left(\int_{0}^{e} \delta \mathrm{r} \times \delta \mathrm{m} d s\right) .
\end{gathered}
$$

We use the above to get the expression for the second variation

$$
\begin{aligned}
D^{2}\left(H+C_{\phi}\right) & =\mathrm{J}^{-1} \delta \mathbf{p} \cdot \delta \mathrm{p}+\int_{0}^{e} \frac{1}{\rho_{0}} \delta \mathrm{m} \cdot \delta \mathrm{m} d s+\int_{0}^{e} \mathbf{K} \frac{\partial \delta \mathrm{r}}{\partial s} \cdot \frac{\partial \delta \mathrm{r}}{\partial s} d s \\
& +2 \phi^{\prime \prime}\left(\|\alpha\|^{2}\right)\left(\alpha \cdot\left(\delta \mathrm{p}+\int_{0}^{e} \mathrm{r} \times \delta \mathrm{m} d s+\int_{0}^{e} \delta \mathrm{r} \times \mathbf{m} d s\right)\right)^{2} \\
& +\phi^{\prime}\left(\|\alpha\|^{2}\right)\left\{\left\|\delta \mathrm{p}+\int_{0}^{e} \mathbf{r} \times \delta \mathrm{m} d s+\int_{0}^{e} \delta \mathrm{r} \times \mathbf{m} d s\right\|^{2}\right. \\
& \left.+2\left(\mathrm{p}+\int_{0}^{e} \mathrm{r} \times \mathrm{m} d s\right) \cdot\left(\int_{0}^{e} \delta \mathrm{r} \times \delta \mathrm{m} d s\right)\right\}
\end{aligned}
$$


258

T. Posbergh, P.S. Krishnaprasad and J. Marsden

3 Computation of a Stability Criterion

The conditions which assure that the first variation $D\left(H+\mathrm{C}_{\phi}\right)$ at an equilibrium is zero are

$$
\begin{aligned}
\phi^{\prime}\left(\left\|\alpha^{e}\right\|^{2}\right) \alpha^{e} & =-\omega^{e} \\
\phi^{\prime}\left(\left\|\alpha^{e}\right\|^{2}\right) \alpha^{e} \times x^{e} & =-\frac{1}{\rho_{0}} \mathbf{m}^{e} \\
\phi^{\prime}\left(\left\|\alpha^{e}\right\|^{2}\right) \alpha^{e} \times m^{e} & =-\mathbf{K} \frac{\partial^{2} \mathbf{r}^{e}}{\partial s^{2}}
\end{aligned}
$$

where $\omega^{e}=J^{-1} p^{e}$, and

$$
\alpha^{e}=\mathbf{p}^{e}+\int_{0}^{e} \mathbf{r}^{e} \times \mathbf{m}^{e} d s .
$$

We use the superscript $e$ to denote evaluation at an equilibrium. If we dot (24) with $\alpha^{e}$ we have

$$
\phi^{\prime}\left(\left\|\alpha^{e}\right\|^{2}\right)=-\frac{\omega^{e} \cdot \alpha^{e}}{\left\|\alpha^{e}\right\|^{2}} .
$$

If we evaluate the first variation at an equilibrium, incorporating the above, then we can derive conditions which assure the stability of the equilibrium. In the following sequence of steps we demonstrate how this is done.

Step 1: Evaluate the Second Variation at an Equilibrium

Recall the second variation. If we use the above to substitute for $\phi^{\prime}\left(\left\|\alpha^{e}\right\|^{2}\right)$ in this expression and rearrange slightly we find that

$$
\begin{aligned}
D^{2}(H & \left.+C_{\phi}\right)_{\left(p^{e}, r^{e}, m^{e}\right)}=\mathbf{J}^{-1} \delta \mathbf{p} \cdot \delta \mathrm{p}+\int_{0}^{l} \frac{1}{\rho_{0}} \delta \mathrm{m} \cdot \delta \mathrm{m} d s \\
& +\int_{0}^{e} \mathbf{K} \frac{\partial \delta \mathbf{r}}{\partial s} \cdot \frac{\partial \delta \mathbf{r}}{\partial s} d s \\
& -\frac{\omega^{e} \cdot \alpha^{e}}{\left\|\alpha^{e}\right\|^{2}}\left\|\delta \mathbf{p}+\int_{0}^{e} \mathbf{r}^{e} \times \delta \mathbf{m} d s+\int_{0}^{e} \delta \mathbf{r} \times \mathbf{m}^{e} d s\right\|^{2} \\
& -2 \frac{\omega^{e} \cdot \alpha^{e}}{\left\|\alpha^{e}\right\|^{2}} \alpha^{e} \cdot\left(\int_{0}^{e} \delta \mathbf{r} \times \delta \mathbf{m} d s\right) \\
& +2 \phi^{\prime \prime}\left(\left\|\alpha^{e}\right\|^{2}\right)\left(\alpha^{e} \cdot\left(\delta \mathbf{p}+\int_{0}^{e} \mathbf{r}^{e} \times \delta \mathbf{m} d s+\int_{0}^{e} \delta \mathbf{r} \times \mathbf{m}^{e} d s\right)\right)^{2},
\end{aligned}
$$


which corresponds to expression (5.5) in Krishnaprasad and Marsden [2]. In that paper, $\phi$ is required to satisfy the condition:

$$
\phi^{\prime \prime}\left(\left\|\alpha^{e}\right\|^{2}\right)=\frac{\omega^{e} \cdot \alpha^{e}}{2\left\|\alpha^{e}\right\|^{4}}
$$

which is consistent with (28). In the following development we impose no conditions on $\phi^{\prime \prime}\left(\left\|\alpha^{e}\right\|^{2}\right)$ at this time.

\section{Step 2: Expand Terms Containing $\delta p$}

We first note that the fourth and sixth terms in (29) can be expanded. For the fourth term we have

$$
\begin{aligned}
-\frac{\omega^{e} \cdot \alpha^{e}}{\left\|\alpha^{e}\right\|^{2}} \| \delta \mathbf{p} & +\int_{0}^{e} s^{e} \times \delta \mathbf{m} d s+\int_{0}^{e} \delta \mathbf{r} \times \mathbf{m}^{e} d s \|^{2} \\
& =-\frac{\omega^{e} \cdot \alpha^{e}}{\left\|\alpha^{e}\right\|^{2}} \delta \mathbf{p} \cdot \delta \mathbf{p} \\
& -2 \frac{\omega^{e} \cdot \alpha^{e}}{\left\|\alpha^{e}\right\|^{2}} \delta \mathbf{p} \cdot\left(\int_{0}^{e} \mathbf{r}^{e} \times \delta \mathrm{m} d s+\int_{0}^{e} \delta \mathbf{r} \times \mathbf{m}^{e} d s\right) \\
& -\frac{\omega^{e} \cdot \alpha^{e}}{\left\|\alpha^{e}\right\|^{2} \|} \int_{0}^{e} \mathbf{r}^{e} \times \delta \mathrm{m} d s+\int_{0}^{e} \delta \mathbf{r} \times \mathbf{m}^{e} d s \|^{2}
\end{aligned}
$$

while for the sixth term

$$
\begin{aligned}
& 2 \phi^{\prime \prime}\left(\left\|\alpha^{e}\right\|^{2}\right)\left(\alpha^{e} \cdot\left(\delta \mathbf{p}+\int_{0}^{e} \mathbf{r}^{e} \times \mathbf{m} d s+\int_{0}^{l} \delta \mathbf{r} \times \mathrm{m}^{e} d s\right)\right)^{2} \\
& \quad=2 \phi^{\prime \prime}\left(\left\|\alpha^{e}\right\|^{2}\right)\left(\alpha^{e} \cdot \delta \mathbf{p}\right)^{2} \\
& \quad+4 \phi^{\prime \prime}\left(\left\|\alpha^{e}\right\|^{2}\right)\left(\alpha^{e} \cdot \delta \mathrm{p}\right)\left(\alpha^{e} \cdot\left(\int_{0}^{e} \mathbf{r}^{e} \times \delta \mathrm{m} d s+\int_{0}^{e} \delta \mathrm{r} \times \mathrm{m}^{e} d s\right)\right) \\
& \quad+2 \phi^{\prime \prime}\left(\left\|\alpha^{e}\right\|^{2}\right)\left(\alpha^{e} \cdot\left(\int_{0}^{e} \mathbf{r}^{e} \times \delta \mathrm{m} d s+\int_{0}^{e} \delta \mathbf{r} \times \mathbf{m}^{e} d s\right)\right)^{2}
\end{aligned}
$$

Step 9: Collect Terms Containing $\delta p$

Now, collect together terms in which the quantity $\delta \mathbf{p}$ appears. Our expression for the second variation at an equilibrium can then be written

$$
\begin{aligned}
& D^{2}\left(H+C_{\phi}\right)= \\
& \quad\left[\mathbf{J}^{-1} \delta \mathbf{p} \cdot \delta \mathbf{p}-\frac{\omega^{e} \cdot \alpha^{e}}{\left\|\alpha^{e}\right\|^{2}}\left(\delta \mathbf{p} \cdot \delta \mathbf{p}+2 \delta \mathbf{p} \cdot\left(\int_{0}^{\ell} \mathbf{r}^{e} \times \delta \mathbf{m} d s+\int_{0}^{\ell} \delta \mathbf{r} \times \mathbf{m}^{e} d s\right)\right)\right.
\end{aligned}
$$




$$
\begin{aligned}
& +2 \phi^{\prime \prime}\left(\left\|\alpha^{e}\right\|^{2}\right)\left\{\left(\alpha^{e} \cdot \delta \mathbf{p}\right)^{2}+2\left(\alpha^{e} \cdot \delta \mathbf{p}\right)\right. \\
& \left.\left.\cdot\left(\alpha^{e} \cdot\left(\int_{0}^{e} \mathbf{r}^{e} \times \delta \mathrm{m} d s+\int_{0}^{e} \delta \mathbf{r} \times \mathrm{m}^{e} d s\right)\right)\right\}\right] \\
& -\frac{\omega^{e} \cdot \alpha^{e}}{\left\|\alpha^{e}\right\|^{2}}\left\|\int_{0}^{e} \mathbf{r}^{e} \times \delta \mathrm{m} d s+\int_{0}^{e} \delta \mathbf{r} \times \mathbf{m}^{e} d s\right\|^{2} \\
& +2 \phi^{\prime \prime}\left(\left\|\alpha^{e}\right\|^{2}\right)\left(\alpha^{e} \cdot\left(\int_{0}^{e} \mathbf{r}^{e} \times \delta \mathrm{m} d s+\int_{0}^{e} \delta \mathbf{r} \times \mathbf{m}^{e} d s\right)\right)^{2} \\
& -2 \frac{\omega^{e} \cdot \alpha^{e}}{\left\|\alpha^{e}\right\|^{2}} \alpha^{e} \cdot\left(\int_{0}^{e} \delta \mathbf{r} \times \delta \mathbf{m} d s\right)+\int_{0}^{e} \frac{1}{\rho_{0}} \delta \mathbf{m} \cdot \delta \mathbf{m} d s+\int_{0}^{l} \mathbf{K} \frac{\partial \delta \mathbf{r}}{\partial s} \cdot \frac{\partial \delta \mathbf{r}}{\partial s} d s .
\end{aligned}
$$

\section{Step 4: Complete the Square}

The term in square brackets which contains the $\delta p$ terms can be rewritten

$$
\begin{aligned}
& {[\cdot]=\left(J^{-1}-\frac{\omega^{e} \cdot \alpha^{e}}{\left\|\alpha^{e}\right\|^{2}} I+2 \phi^{\prime \prime}\left(\left\|\alpha^{e}\right\|^{2}\right) \alpha^{e} \otimes \alpha^{e}\right) \delta p \cdot \delta p} \\
& ,+2\left(-\frac{\omega^{e} \cdot \alpha^{e}}{\left\|\alpha^{e}\right\|^{2}} I+2 \phi^{\prime \prime}\left(\left\|\alpha^{e}\right\|^{2}\right) \alpha^{e} \otimes \alpha^{e}\right) \delta p \\
& \cdot\left(\int_{0}^{e} \mathrm{r}^{e} \times \delta \mathrm{m} d s+\int_{0}^{e} \delta \mathrm{r} \times \mathrm{m}^{e} d s\right) .
\end{aligned}
$$

In this expression we use $\otimes$ to denote the tensor product and $I$ the identity. Note that $\alpha^{e} \otimes \alpha^{e}$ is a tensor of rank 2. We can complete the square for this expression provided the quantity

$$
J^{-1}-\frac{\omega^{e} \cdot \alpha^{e}}{\left\|\alpha^{e}\right\|^{2}} I+2 \phi^{\prime \prime}\left(\left\|\alpha^{e}\right\|^{2}\right) \alpha^{e} \otimes \alpha^{e}
$$

has an inverse.

We next assume this inverse exists and define the two symmetric matrices $M$ and $N$ by,

$$
\begin{aligned}
M^{T} M & =J^{-1}-\frac{\omega^{e} \cdot \alpha^{e}}{\left\|\alpha^{e}\right\|^{2}} I+2 \phi^{\prime \prime}\left(\left\|\alpha^{e}\right\|^{2}\right) \alpha^{e} \otimes \alpha^{e} \\
& \triangleq J_{e}^{-1} \\
N^{T} M & =-\frac{\omega^{e} \cdot \alpha^{e}}{\left\|\alpha^{e}\right\|^{2}} I+2 \phi^{\prime \prime}\left(\left\|\alpha^{e}\right\|^{2}\right) \alpha^{e} \otimes \alpha^{e}
\end{aligned}
$$




$$
\triangleq \mathbf{Q}_{\mathbf{e}}
$$

Completing the square for the term in brackets we now get

$$
\begin{aligned}
& {[\cdot]=\left\|\mathbf{M} \delta p+\mathbf{N}\left(\int_{0}^{e} \mathbf{r}^{e} \times \delta \mathbf{m} d s+\int_{0}^{e} \delta \mathbf{r} \times \mathbf{m}^{e} d s\right)\right\|^{2}} \\
& -\mathrm{N}^{T} \mathrm{~N}\left(\int_{0}^{e} \mathrm{r}^{e} \times \delta \mathrm{m} d \mathrm{~s}+\int_{0}^{e} \delta \mathrm{r} \times \mathrm{m}^{e} d s\right) \\
& \cdot\left(\int_{0}^{\ell} \mathrm{r}^{e} \times 6 \mathrm{~m} d s+\int_{0}^{\ell} \delta \mathrm{r} \times \mathrm{m}^{e} d s\right) \text {. }
\end{aligned}
$$

The term in braces is bounded below by a perfect square when $N^{T} N \geq 0$. For this to be the case we need to assume that the inverted matrix, $J_{e}^{-1}$ is positive definite, in general it need not be. Note that this assumption will impose conditions on $\phi^{\prime \prime}\left(\left\|\alpha^{e}\right\|^{2}\right)$. The requirements on the parameters in this matrix to assure it is strictly positive definite will be expressed in the form of inequalities. These inequalities will be the first conditions that we need to assure stability.

\section{Step 5: The Reformulated Second Variation}

The second variation at an equilibrium is thus of the form

$$
\begin{aligned}
& D^{2}\left(H+C_{\phi}\right)=(\text { square }) \\
& -\mathbf{N}^{T} \mathbf{N}\left(\int_{0}^{e} \mathbf{r}^{e} \times \delta \mathrm{m} d s+\int_{0}^{e} \delta \mathbf{r} \times \mathrm{m}^{e} d s\right) \\
& \cdot\left(\int_{0}^{e} \mathbf{r}^{e} \times \delta \mathrm{m} d s+\int_{0}^{e} \delta \mathrm{r} \times \mathrm{m}^{e} d s\right) \\
& \left.-\frac{\omega^{e} \cdot \alpha^{e}}{\left\|\alpha^{e}\right\|^{2}} \| \int_{0}^{e} r^{e} \times \delta \mathrm{m} d s+\int_{0}^{e} \delta \mathrm{r} \times \mathrm{m}^{e} d s\right) \|^{2} \\
& +2 \phi^{\prime \prime}\left(\left\|\alpha^{e}\right\|^{2}\right) \alpha^{e} \otimes \alpha^{e}\left(\int_{0}^{e} \mathbf{r}^{e} \times \delta \mathrm{m} d s+\int_{0}^{e} \delta r \times \mathrm{m}^{e} d s\right) \\
& \cdot\left(\int_{0}^{e} r^{e} \times \delta \mathrm{m} d s+\int_{0}^{e} \delta \mathrm{r} \times \mathrm{m}^{e} d s\right) \\
& -2 \frac{\omega^{e} \cdot \alpha^{e}}{\left\|\alpha^{e}\right\|^{2}} \alpha^{e} \cdot\left(\int_{0}^{e} \delta \mathbf{r} \times \delta \mathbf{m} d s\right) \\
& +\int_{0}^{\ell} \frac{1}{\rho_{0}} \delta \mathrm{m} \cdot \delta \mathbf{m} d s+\int_{0}^{\ell} \mathbf{K} \frac{\partial \delta x}{\partial s} \cdot \frac{\partial \delta \mathbf{r}}{\partial s} d s .
\end{aligned}
$$


Where we note that

$$
\begin{aligned}
N^{T} N & =\left(-\frac{\omega^{e} \cdot \alpha^{e}}{\left\|\alpha^{e}\right\|^{2}} I+2 \phi^{\prime \prime}\left(\left\|\alpha^{e}\right\|^{2}\right) \alpha^{e} \otimes \alpha^{e}\right) \\
& \left(J^{-1}-\frac{\omega^{e} \cdot \alpha^{e}}{\left\|\alpha^{e}\right\|^{2}} I+2 \phi^{\prime \prime}\left(\left\|\alpha^{e}\right\|^{2}\right) \alpha^{e} \otimes \alpha^{e}\right)^{-1} \\
& \left(-\frac{\omega^{e} \cdot \alpha^{e}}{\left\|\alpha^{e}\right\|^{2}} I+2 \phi^{\prime \prime}\left(\left\|\alpha^{e}\right\|^{2}\right) \alpha^{e} \otimes \alpha^{e}\right) \\
& =Q_{e} J_{e} Q_{e}
\end{aligned}
$$

\section{Step 6: Collect Integrals of Cross Products}

Collecting terms containing the integrals of cross products the second variation can be written

$$
\begin{aligned}
& D^{2}\left(H+C_{\phi}\right)=\text { (square) } \\
& -\left(Q_{e} J_{e} Q_{e}-Q_{e}\right)\left(\int_{0}^{e} r^{e} \times \delta \mathrm{m} d s+\int_{0}^{e} \delta \mathrm{r} \times \mathrm{m}^{e} d s\right) \\
& -\left(\int_{0}^{e} \mathbf{r}^{e} \times \delta \mathrm{m} d s+\int_{0}^{e} \delta \mathrm{r} \times \mathrm{m}^{e} d s\right) \\
& -2 \frac{\omega^{e} \cdot \alpha^{e}}{\left\|\alpha^{e}\right\|^{2}} \alpha^{e} \cdot\left(\int_{0}^{e} \delta \mathrm{r} \times \delta \mathrm{m} d s\right)+ \\
& \int_{0}^{e} \frac{1}{\rho_{0}} \delta \mathrm{m} \times \delta \mathrm{m} d s+\int_{0}^{e} \mathbf{K} \frac{\partial \delta \mathrm{r}}{\partial s} \cdot \frac{\partial \delta \mathbf{r}}{\partial s} d s
\end{aligned}
$$

\section{Step 7: A Vector Identity}

Observe that a simple vector identity enables us to write

$$
\begin{aligned}
2 \frac{\omega^{e} \cdot \alpha^{e}}{\left\|\alpha^{e}\right\|^{2}} \alpha^{e} \cdot\left(\int_{0}^{e} \delta \mathbf{r} \times \delta \mathrm{m} d s\right) & =\int_{0}^{e}\left(\frac{\alpha^{e} \cdot \omega^{e}}{\left\|\alpha^{e}\right\|^{2}} \alpha^{e} \times \delta \mathbf{r}\right) \cdot \delta \mathrm{m} d s \\
& =2 \int_{0}^{e} \delta \mathrm{m}^{T} S\left(\frac{\left(\alpha^{e} \otimes \alpha^{e}\right) \omega^{e}}{\left\|\alpha^{e}\right\|^{2}}\right) \delta \mathbf{r} d s
\end{aligned}
$$

where we have used the skew-symmetric matrix $S(x)$ associated with the crossproduct

$$
S(x)=\left[\begin{array}{ccc}
0 & -x_{3} & x_{2} \\
x_{3} & 0 & -x_{1} \\
-x_{2} & x_{1} & 0
\end{array}\right]
$$


Step 8: A Quadratic Form

Now define the symmetric matrix

$$
\mathbf{R} \triangleq \mathbf{Q}_{e} \mathbf{J}_{\mathbf{e}} \mathbf{Q}_{\boldsymbol{e}}-\mathbf{Q}_{\boldsymbol{e}} \text {. }
$$

We will see below, that an eigenvalue estimate (46) relies on having $R$ nonnegative definite. We thus require that conditions on the parameters of the problem and $\phi^{\prime \prime}\left(\left\|\alpha^{e}\right\|^{2}\right)$ hold such that $J_{e}^{-1}$ defined in (36) is positive definite and $\mathbf{R}$ defined in (44) is nonnegative definite. The latter will assure that $R$ has a square root $R^{1 / 2}$. We will examine these assumptions again in remark 2 below.

Expanding the second term in (41), we can re-express it as a quadratic form,

$$
\begin{aligned}
\mathbf{R}\left(\int_{0}^{e} \mathbf{r}^{e} \times \delta \mathbf{m} d s+\int_{0}^{e} \delta \mathbf{r} \times \mathbf{m}^{e} d s\right) \cdot\left(\int_{0}^{e} x^{e} \times \delta \mathbf{m} d s+\int_{0}^{e} \delta \mathbf{r} \times \mathbf{m}^{e} d s\right) \\
=\int_{0}^{e} \int_{0}^{e} \mathbf{R}\left(\mathbf{S}\left(\mathbf{r}^{e}(s)\right) \delta \mathbf{m}(s)-\mathbf{S}\left(\mathbf{m}^{e}(s)\right) \delta \mathbf{r}(s)\right) \\
\cdot\left(\mathbf{S}\left(\mathbf{r}^{e}(\sigma)\right) \delta \mathbf{m}(\sigma)-\mathbf{S}\left(\mathbf{m}^{e}(\sigma)\right) \delta \mathbf{r}(\sigma)\right) d s d \sigma \\
=\int_{0}^{e} \int_{0}^{l}\left[\delta \mathbf{m}^{T}(s) \delta \mathbf{r}^{T}(s)\right]\left[\begin{array}{c}
\mathbf{S}^{T}\left(\mathbf{r}^{e}(s)\right) \\
-\mathbf{S}^{T}\left(\mathbf{m}^{e}(s)\right)
\end{array}\right] \\
\mathbf{R}\left[\mathbf{S}\left(\mathbf{r}^{e}(\sigma)\right)-\mathbf{S}\left(\mathbf{m}^{e}(\sigma)\right)\right]\left[\begin{array}{c}
\delta \mathbf{m}(\sigma) \\
\delta \mathbf{r}(\sigma)
\end{array}\right] d s d \sigma \\
=\int_{0}^{e} \int_{0}^{e}\left[\delta \mathbf{m}^{T}(s) \delta \mathbf{r}^{T}(s)\right] \mathbf{A}^{T}(s) \mathbf{A}(\sigma)\left[\begin{array}{c}
\delta \mathbf{m}(\sigma) \\
\delta \mathbf{r}(\sigma)
\end{array}\right] d s d \sigma
\end{aligned}
$$

We now can find a lower bound on the above. The bound we want is obtained from an eigenvalue inequality which we introduce by way of the following lemma.

Step 9: An Eigenvalue Inequality

Lemma 3.1 : Let $\mathbf{A}(s) \in L_{2}^{n \times n}(0, \ell)$, and $\mathbf{x}(s) \in L_{2}^{n}(0, \ell)$ then

$$
\int_{0}^{e} \int_{0}^{e} x^{T}(s) A^{T}(s) A(\sigma) x(\sigma) d \sigma d s \leq \int_{0}^{e} x^{T}(s)\left\{\int_{0}^{e} I \lambda^{2}(\sigma) d \sigma\right\} x(s) d s,
$$

where $\lambda^{2}(s)$ is the maximum eigenvalue of $A^{T}(s) A(s)$.

Proof: Let $\|$. \| denote the standard norm in Euclidean space and also the induced matrix norm associated with it. Then

$$
\int_{0}^{e} \int_{0}^{l} x^{T}(s) A^{T}(s) A(\sigma) x(\sigma) d \sigma d s
$$




$$
\begin{aligned}
& \leq \int_{0}^{e} \int_{0}^{l} \mid \mathbf{x}^{T}(s) A^{T}(s) A(\sigma) x(\sigma) \| d \sigma d s \\
& \leq \int_{0}^{e} \int_{0}^{l}\|A(s) x(s)\|\|A(\sigma) x(\sigma)\| d \sigma d s \\
& \leq \int_{0}^{e}\|A(s)\|\|x(s)\| d s \int_{0}^{e}\|A(\sigma)\|\|x(\sigma)\| d \sigma
\end{aligned}
$$

where we have used $\|A(s) x(s)\| \leq\|A(s)\|\|x(s)\|$. We can now use the Schwarz inequality

$$
\left(\int_{0}^{l}\|\mathbf{A}(s)\|\|\mathbf{x}(s)\| d s\right)^{2} \leq \int_{0}^{e}\|\mathbf{A}(s)\|^{2} d s \int_{0}^{e}\|\mathbf{x}(s)\|^{2} d s .
$$

Finally noting that the value of $\|A(s)\|$ is simply the square root of the maximum eigenvalue of $A^{T}(s) A(s)$ establishes the result.

If we let $\lambda^{2}(s)$ be the maximum eigenvalue of

$$
\mathbf{A}^{T}(s) A(s)=\left[\begin{array}{cc}
\mathbf{S}^{T}\left(\mathbf{r}^{e}(s)\right) \mathbf{R S}\left(\mathbf{r}^{e}(s)\right) & -\mathbf{S}^{T}\left(\mathbf{r}^{e}(s)\right) \mathbf{R S}\left(\mathbf{m}^{e}(s)\right) \\
-\mathbf{S}^{T}\left(\mathbf{m}^{e}(s)\right) \mathbf{R S}\left(\mathbf{r}^{e}(s)\right) & \mathbf{S}^{T}\left(\mathbf{m}^{e}(s)\right) \mathbf{R S}\left(\mathbf{m}^{e}(s)\right)
\end{array}\right]
$$

and let $\bar{\lambda}^{2}=\int_{0}^{l} \lambda^{2}(s) d s$ then we have by way of lemma 3.1 a lower bound on the second variation

$$
\begin{aligned}
& D^{2}\left(\mathbf{H}+\mathrm{C}_{\phi}\right)_{\left(\mu^{*}, \mathbf{I}^{*}, \mathrm{~m}^{*}\right)} \geq \text { (square) } \\
& -\bar{\lambda}^{2} \int_{0}^{l} \delta \mathbf{m}^{T} \delta \mathbf{m} d s-\bar{\lambda}^{2} \int_{0}^{l} \delta \mathbf{r}^{T} \delta r d s \\
& -2 \int_{0}^{e} \delta m^{T} S\left(\frac{\left(\alpha^{e} \otimes \alpha^{e}\right) \omega^{e}}{\left\|\alpha^{e}\right\|^{2}}\right) \delta r d s \\
& +\int_{0}^{l} \frac{1}{\rho_{0}} \delta \mathbf{m}^{T} \delta \mathrm{m} d s+\int_{0}^{l} \mathrm{~K} \frac{\partial \delta \mathbf{r}}{\partial s} \cdot \frac{\partial \delta \mathbf{r}}{\partial s} d s
\end{aligned}
$$

Step 10: A Poincaré Type Inequality

If we assume that $\mathrm{K}$ is diagonal and use a Poincaré-type inequality

$$
\int_{0}^{\ell} \mathbf{K} \frac{\partial \delta \mathbf{r}}{\partial s} \cdot \frac{\partial \delta \mathbf{r}}{\partial s} d s \geq c \int_{0}^{\ell} \mathbf{K} \delta \mathbf{r} \cdot \delta \mathbf{r} d s_{1}
$$


with $c=\left(\frac{\pi}{2 \ell}\right)^{2}$, then the second variation can be bounded below as

$$
\begin{aligned}
& D^{2}\left(\mathrm{H}+\mathrm{C}_{\phi}\right)_{\left(p^{*}, \mathrm{I}^{*}, \mathrm{~m} \cdot\right)} \geq \text { (oquare) } \\
& -\bar{\lambda}^{2} \int_{0}^{e} \delta m^{T} \delta m d s-\tilde{\lambda}^{2} \int_{0}^{e} \delta r^{T} \delta r d s \\
& -2 \int_{0}^{l} \delta \mathrm{m}^{T} \mathrm{~s}\left(\frac{\left(\alpha^{e} \otimes \alpha^{e}\right) \omega^{e}}{\left\|\alpha^{e}\right\|^{2}}\right) \delta \mathbf{r} d s \\
& +\int_{0}^{l} \frac{1}{\rho_{0}} \delta \mathrm{m}^{T} \delta \mathrm{m} d s+c \int_{0}^{l} \delta \mathrm{r}^{T} \mathrm{~K} \delta \mathrm{x} d s .
\end{aligned}
$$

Step 11: Rewrite The Lower Bound

We can reformulate the lower bound in a clearer form as follows

$$
\begin{aligned}
& D^{2}\left(H+C_{\phi}\right)_{\left(D^{4}, r^{e},\right. \text { mer) }} \geq(\text { square })
\end{aligned}
$$

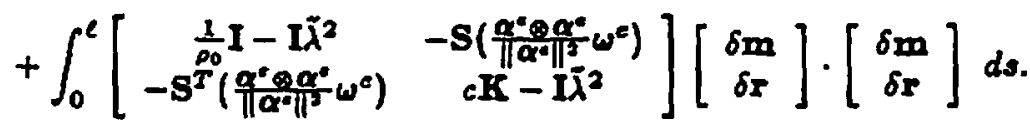

If we define the matrix

$$
D\left(p^{e}, x^{e}, m^{e}\right)=\left[\begin{array}{cc}
\frac{1}{p_{0}} I-I \bar{\lambda}^{2} & -S\left(\frac{\alpha^{e} \alpha \alpha^{e}}{\left\|\alpha^{e}\right\|^{2}} \omega^{e}\right) \\
-S^{T}\left(\frac{\alpha^{e} \alpha \alpha^{*}}{\left\|\alpha^{*}\right\|^{2}} \omega^{c}\right) & c K-I \bar{\lambda}^{2}
\end{array}\right]
$$

then we can state the following theorem;

Theorem 3.1 : If the matriz $\mathbf{R}=\mathbf{Q}_{e} \mathrm{~J}_{e} \mathbf{Q}_{\boldsymbol{e}}-\mathbf{Q}_{\boldsymbol{e}}$ defined in (4f) ezists and is nonnegative definite, $\mathrm{J}_{e}$ defined in (\$6) is positive definite, and the matrix $\mathbf{D}$ defined in equation (52) is positive definite, then the system described by equations (2)-(4) is nonlinearly (formally) stable at the equilibrium point $\left(\mathrm{p}^{e}, \mathrm{x}^{e}, \mathrm{~m}^{e}\right)$.

Remark 1 : This result establishes only formal stability, since it is based on the definiteness of second variational To establish rigorous stability of the nonlinear system one generally needs to examine convexity estimates as is done in [2].

Remark 2 : Note that if $\mathbf{Q}_{e}^{-1}$ exists and we use the matrix inversion lemma [4], p.656, we obtain the following

$$
\begin{aligned}
\left(Q_{e}^{-1}+J^{-1}\right. & =Q_{e}-Q_{e} J_{e} Q_{e} \\
& =-\mathbf{R}
\end{aligned}
$$


Recall that we already have an assumption of nonnegative definileness on $\mathbf{R}$. Thus we need to specify conditions on the parameters and $\phi^{\prime \prime}\left(\left\|\alpha^{e}\right\|^{2}\right)$ such that

$$
\begin{aligned}
\mathbf{J}^{-1}+\mathbf{Q}_{e} & >0 \\
\left(\mathbf{J}+\mathbf{Q}_{e}^{-1}\right)^{-1} & \leq 0
\end{aligned}
$$

which are the same conditions as $\mathrm{R} \geq 0$ and $\mathrm{J}_{e}>0$. In the examples of the next section $\mathbf{Q}_{\boldsymbol{c}}$ is singular.

Remark 3 : $A$ better result can be had by observing that $A^{T}(s) A(s)$ is frequently in the form of a block diagonal matrix

$$
A^{T}(s) A(s)=\left[\begin{array}{ccc}
A_{1}^{T}(s) A_{1}(s) & & 0 \\
0 & \ddots & \\
A_{k}^{T}(s) A_{k}(s)
\end{array}\right]
$$

where $0 \leq k \leq 6$ and because of the semidefiniteness of $A^{T} A(s)$ some of the diagonal blocks may be zero. If we let $\lambda_{i}^{2}(s)$ be the maximum eigenvalue of $A_{i}^{T}(s) A_{i}(s), 0 \leq i \leq k$ then we can define

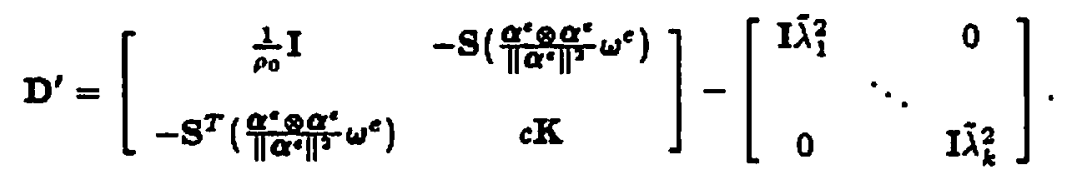

Thus, if the conditions of theorem 3.2 are satisfied and also the matrix $\mathrm{D}^{\prime}$ defined in equation (57) is positive definite, then the system described by equations $(\varepsilon)-(t)$ is (formally) nonlinearly stable at the equilibrium point $\left(\mathrm{p}^{e}, \mathrm{r}^{e}, \mathrm{~m}^{e}\right)$. In theorem $s .2$ this will mean the special choice $\lambda^{2}(s)=\max \left\{\lambda_{1}^{2}(s), \ldots, \lambda_{k}^{2}(s)\right\}$.

\section{Some Examples}

In this section we apply theorem 3.1 to specific equilibria of (2)-(4). We will assume that the linear extensible shear beam lies along the same direction as the second principal axis of inertia of the rigid body. From geometric considerations the position of the shear beam will cause the principal axes of the rigid-bodyshear-beam configuration to lie in the same directions as those of the rigid body. In this case the addition of the shear beam will have the effect of increasing the moments of inertia about the first and the third principal axes. Because the linear extensible shear beam cannot deflect laterally the principal axes of the configuration rémain fixed for any longitudinal extension of the shear beam. Thus, for this configuration there are three axes about which the equilibria can exist. These axes will correspond to the three principal axes of the rigid body. 


\subsection{A Trivial Equilibrium}

The simplest case to be considered is when the rotation takes place about the axis along which the linear extensible shear beam lies. In this case the equilibrium will be

$$
\begin{aligned}
\omega^{e} & =\omega_{2}^{e} \hat{e}_{2} \\
\mathbf{r}^{e} & =\left(a_{2}+s\right) \hat{e}_{2}, \quad 0 \leq s \leq \ell \\
\mathbf{m}^{e} & =0 .
\end{aligned}
$$

This degcribes the linear-extensible-shear-beam being ungtretched.

What follows is a special case of the second variation computed in Step 1 of the previous section. In this and the following example we will assume $\phi^{\prime \prime}\left(\left\|\alpha^{e}\right\|^{2}\right)$ is the same as in [2], thus recall from (30) that if this is the case then

$$
\phi^{\prime \prime}\left(\left\|\alpha^{e}\right\|^{2}\right)=\frac{\omega^{e} \cdot \alpha^{e}}{2\left\|\alpha^{e}\right\|^{4}} .
$$

And the two quantities, $J_{e}^{-1}$, and $Q_{e}$, which we define in Step 4 are

$$
\begin{array}{r}
J_{e}^{-1}=J^{-1}-\frac{\omega^{e} \cdot \alpha^{e}}{\left\|\alpha^{e}\right\|^{2}}\left(I-\frac{\alpha^{e} \otimes \alpha^{e}}{\left\|\alpha^{e}\right\|^{2}}\right) \\
Q_{c}=-\frac{\omega^{e} \cdot \alpha^{e}}{\left\|\alpha^{e}\right\|^{2}}\left(I-\frac{\alpha^{e} \otimes \alpha^{e}}{\left\|\alpha^{e}\right\|^{2}}\right) .
\end{array}
$$

For our example, if we first compute

$$
\alpha^{e}=j_{22} \omega_{2} \hat{e}_{2}
$$

then

$$
\alpha^{e T} \omega^{e}=j_{22}\left(\omega_{2}^{e}\right)^{2} \text { and } \alpha^{e T} \alpha^{e}=j_{22}^{2}\left(\omega_{2}^{e}\right)^{2}
$$

from which we immediately compute

$$
I-\frac{\alpha^{e} \alpha^{e T}}{\alpha^{e T} \alpha^{e}}=\left[\begin{array}{lll}
1 & 0 & 0 \\
0 & 0 & 0 \\
0 & 0 & 1
\end{array}\right]
$$

and finally,

$$
\begin{aligned}
J_{e}^{-1} & =\left[\begin{array}{ccc}
\frac{j j_{22}}{j_{22}-j_{11}} & 0 & 0 \\
0 & j_{22} & 0 \\
0 & 0 & \frac{j_{23} j_{22}}{j_{22}-j_{33}}
\end{array}\right] \\
\mathbf{Q}_{e} & =\left[\begin{array}{ccc}
\frac{1}{j_{22}} & 0 & 0 \\
0 & 0 & 0 \\
0 & 0 & \frac{1}{j_{23}}
\end{array}\right] .
\end{aligned}
$$


For $J_{e}$ to be positive definite we require $j_{22}>j_{11}$, and $j_{22}>j_{33}$. This will assure positive elements along the diagonal in the inverse above.

Thus, the quantity $Q_{e} J_{e} Q_{e}$ which appears in the reformulated second variation of Step 5 will be,

$$
\begin{aligned}
& Q_{e} J_{e} Q_{e}=\left(J^{-1}-\frac{\alpha^{e T} \omega^{e}}{\alpha^{e T} \alpha^{e}}\left(I-\frac{\alpha^{e} \alpha^{e T}}{\alpha^{e T} \alpha^{e}}\right)\right)^{-1}\left(\frac{\left(\alpha^{e T} \omega^{e}\right)^{2}}{\left(\alpha^{e T} \alpha^{e}\right)^{2}}\left(I-\frac{\alpha^{e} \alpha^{e T}}{\alpha^{e T} \alpha^{e}}\right)\right) \\
& =\left(\left[\begin{array}{ccc}
\frac{1}{j_{11}} & 0 & 0 \\
0 & \frac{1}{j_{22}} & 0 \\
0 & 0 & \frac{1}{j_{33}}
\end{array}\right]-\left[\begin{array}{ccc}
\frac{1}{j_{22}} & 0 & 0 \\
0 & 0 & 0 \\
0 & 0 & \frac{1}{j_{32}}
\end{array}\right]\right)^{-1} \\
& \left(\left[\begin{array}{ccc}
\frac{1}{j_{23}^{2}} & 0 & 0 \\
0 & 0 & 0 \\
0 & 0 & \frac{1}{j_{32}^{2}}
\end{array}\right]\right) \\
& =\left[\begin{array}{ccc}
\frac{j_{11} j_{23}}{j_{23}-j_{11}} & 0 & 0 \\
0 & j_{22} & 0 \\
0 & 0 & \frac{j_{23} j_{23}}{j_{32}-j_{32}}
\end{array}\right]\left[\begin{array}{ccc}
\frac{1}{j_{22}^{2}} & 0 & 0 \\
0 & 0 & 0 \\
0 & 0 & \frac{1}{j_{32}^{j}}
\end{array}\right] \\
& =\left[\begin{array}{ccc}
\frac{j_{11}}{j_{23}\left(j_{22}-j_{11}\right)} & 0 & 0 \\
0 & 0 & 0 \\
0 & 0 & \frac{j_{13}}{j_{22}\left(j_{23}-j_{33}\right)}
\end{array}\right]
\end{aligned}
$$

where we have used equation (40) and the fact that $J_{e}$ and $Q_{e}$ are diagonal.

We also need the skew symmetric matrix which appears in Step 7. Thus, we compute

$$
\mathbf{S}\left(\frac{\alpha^{e} \alpha^{e T}}{\alpha^{e T} \alpha^{e}} \omega^{e}\right)=\left[\begin{array}{ccc}
0 & 0 & \omega_{2}^{e} \\
0 & 0 & 0 \\
-\omega_{2}^{e} & 0 & 0
\end{array}\right] .
$$

Now we compute $\mathbf{R}$, which is defined in Step 8 .

$$
\begin{aligned}
& \mathbf{R}=Q_{e} J_{e} Q_{e}+\frac{\alpha^{e T} \omega^{e}}{\alpha^{e T} \alpha^{e}}\left(I-\frac{\alpha^{e} \alpha^{e T}}{\alpha^{e T} \alpha^{e}}\right) \\
& =\left[\begin{array}{ccc}
\frac{j}{j_{23}\left(j j_{23}-j_{11}\right)} & 0 & 0 \\
0 & 0 & 0 \\
0 & 0 & \frac{j}{j_{32}\left(j_{23}-j_{33}\right)}
\end{array}\right]+\left[\begin{array}{ccc}
\frac{1}{j_{j 2}} & 0 & 0 \\
0 & 0 & 0 \\
0 & 0 & \frac{1}{j_{23}}
\end{array}\right] \\
& =\left[\begin{array}{ccc}
\frac{1}{j_{22}-j_{11}} & 0 & 0 \\
0 & 0 & 0 \\
0 & 0 & \frac{1}{j_{22}-j_{39}}
\end{array}\right]
\end{aligned}
$$


which, along with the definition of $S(\cdot)$ in (43), we can now use to compute

$$
\begin{aligned}
\mathbf{S}^{T}\left(\mathbf{r}^{e}\right) \mathbf{R S}\left(\mathbf{r}^{e}\right) & =\left[\begin{array}{ccc}
\frac{1}{j_{32}-j_{33}} r_{2}^{e 2} & 0 & 0 \\
0 & 0 & 0 \\
0 & 0 & \frac{1}{j 32-j_{12}} r_{2}^{e 2}
\end{array}\right] \\
\mathbf{S}^{T}\left(\mathbf{r}^{e}\right) \mathbf{R S}\left(\mathbf{m}^{e}\right) & =0 \\
\mathbf{S}^{T}\left(\mathbf{m}^{e}\right) \mathbf{R S}\left(\mathbf{m}^{e}\right) & =0 .
\end{aligned}
$$

These matrices are used to form the matrix $A^{T}(s) A(s)$ in (57), note that it has only the two nonsero elements (computed in (72)). These correspond to the firat and second diagonal elements. Hence, $A^{T}(s) A(s)$ is a diagonal matrix and the nonsero eigenvalues are these two elements. As a consequence we will use the modified bound described in Remark 9 . Thus, the eigenvalue inequality is easily obtained.

After using the Poincare inequality of Step 10 we proceed to the final step and construct the $D^{\prime}$ matrix in (57)

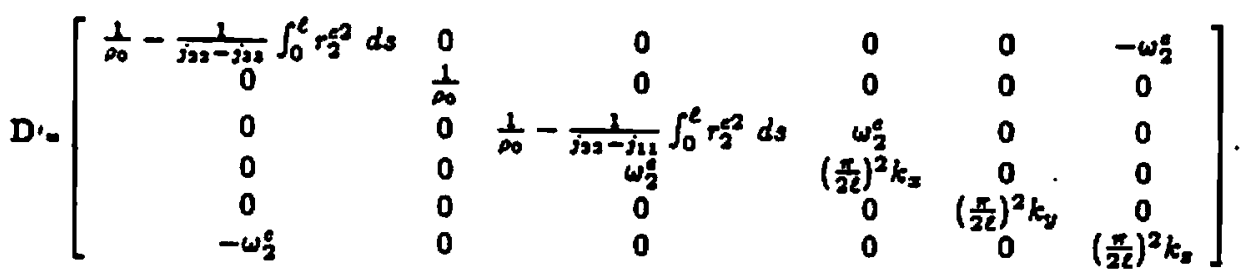

To assure that the $\mathbf{D}^{\prime}$ matrix is positive definite we require

$$
\begin{aligned}
& j_{22}-j_{11}>\rho_{0} \int_{0}^{e} r_{2}^{e 2} d s \\
& j_{22}-j_{33}>\rho_{0} \int_{0}^{e} r_{2}^{e 2} d s
\end{aligned}
$$

and also,

$$
\begin{aligned}
& \left(\frac{1}{\rho_{0}}-\frac{1}{j_{22}-j_{33}} \int_{0}^{\ell} r_{2}^{e 2} d s\right)\left(\frac{\pi}{2 \ell}\right)^{2} k_{z}>\left(\omega_{2}^{e}\right)^{2} \\
& \left(\frac{1}{\rho_{0}}-\frac{1}{j_{22}-j_{11}} \int_{0}^{\ell} r_{2}^{e 2} d s\right)\left(\frac{\pi}{2 \ell}\right)^{2} k_{x}>\left(\omega_{2}^{e}\right)^{2} .
\end{aligned}
$$


Physically the first two conditions are classical stability conditions on the stable axes of rotation for a rigid body. The term on the right is the additional inertia due to the flexible appendage which adds inertia about both the first and third axes. The second two inequalities are conditions on the admissible rotation rates of the configuration. They have an interesting physical interpretation.

\subsection{A Non-Trivial Equilibrium}

For the second example we will consider rotations of the rigid-body-shear-beam configuration about the first or third principal axes of inertia. We will examine the case when the rotation is about the first principal axis of inertia, rotations about the third axis are similar. This corresponds to the example in Krishnaprasad and Marsden [2].

$$
\begin{aligned}
\omega^{e} & =\omega_{1}^{e} \hat{e}_{1} \\
\mathbf{r}^{e}(s) & =\left(\frac{\sin \left(\sqrt{\frac{\rho_{0}}{k_{y}}} \omega_{1}^{e} s\right)}{\sqrt{\frac{\rho_{0}}{k_{y}}} \omega_{1}^{e}}+a \frac{\cos \left(\sqrt{\frac{\rho_{0}}{k_{y}}} \omega_{1}^{e}(s-\ell)\right)}{\cos \left(\sqrt{\frac{\rho_{0}}{k_{y}}} \omega_{1}^{e} \ell\right)}\right) \hat{\mathbf{e}}_{2} \\
\mathbf{m}^{e}(s) & =\rho_{0} \omega_{1}\left(\frac{\sin \left(\sqrt{\frac{\rho_{0}}{k_{y}}} \omega_{1}^{e} s\right)}{\sqrt{\frac{\rho_{0}}{k_{y}}} \omega_{1}^{e}}+a \frac{\cos \left(\sqrt{\frac{\rho_{0}}{k_{y}}} \omega_{1}^{e}(s-\ell)\right)}{\cos \left(\sqrt{\frac{\rho_{0}}{k_{y}}} \omega_{1}^{e} \ell\right)}\right) \hat{\mathbf{e}}_{3}
\end{aligned}
$$

In these equations we have $0 \leq s \leq \ell$. For simplicity we will denote the nonzero element of $r$ as $r_{2}^{e}$, and that of $m$ as $m_{3}^{e}$.

We first compute

$$
\alpha^{e}=j_{11} \omega_{1}^{e}+\int_{0}^{\ell} r_{2}^{e} \mathbf{m}_{3}^{e} d s \hat{\mathbf{e}}_{1}
$$

thus

$$
\begin{aligned}
& \alpha^{e T} \omega^{e}=\left(j_{11} \omega_{1}^{e}+\int_{0}^{l} r_{2}^{e} m_{3}^{e} d s\right) \omega_{1}^{e} \\
& \alpha^{e T} \alpha^{e}=\left(j_{11} \omega_{1}^{e}+\int_{0}^{l} r_{2}^{e} m_{3}^{e} d s\right)^{2} .
\end{aligned}
$$

Subsequently we will denote the first element of $\alpha$ by $\alpha_{1}$. We now compute

$$
I-\frac{\alpha^{e} \alpha^{e T}}{\alpha^{e T} \alpha^{e}}=\left[\begin{array}{lll}
0 & 0 & 0 \\
0 & 1 & 0 \\
0 & 0 & 1
\end{array}\right]
$$


and finally, $\mathrm{J}_{e}^{-1}$ and $\mathbf{Q}_{e}$ defined in Step $\&$ are

$$
\begin{aligned}
J_{e}^{-1} & =\left[\begin{array}{ccc}
\frac{1}{j_{11}} & 0 & 0 \\
0 & \frac{\alpha_{2}-j_{22} \omega_{i}^{*}}{j_{32} \alpha_{1}} & 0 \\
0 & 0 & \frac{\alpha_{1}-j_{33} \omega_{i}^{*}}{j_{33} \alpha_{1}}
\end{array}\right] \\
Q_{e} & =\left[\begin{array}{ccc}
0 & 0 & 0 \\
0 & \frac{\omega_{i}^{i}}{\alpha_{1}} & 0 \\
0 & 0 & \frac{\omega_{i}^{i}}{\alpha_{1}}
\end{array}\right] .
\end{aligned}
$$

For $\mathrm{J}_{e}^{-1}$ to be positive definite we require

$$
\alpha_{1}>j_{22} \omega_{1}^{e} \text { and } \alpha_{1}>j_{33} \omega_{1}^{e} \text {. }
$$

These conditions will hold if $j_{11}>j_{22}$, and $j_{11}>j_{33}$ and will assure positive elements along the diagonal in the inverse above. These conditions are the game as $(5.10)$ in $(2)$.

Then from equation (40) we have

$$
\begin{aligned}
& Q_{e} J_{e} Q_{e}=\left(J^{-1}-\frac{\alpha^{e T} \omega^{e}}{\alpha^{e T} \alpha^{e}}\left(I-\frac{\alpha^{e} \alpha^{e T}}{\alpha^{e T} \alpha^{e}}\right)\right)^{-1}\left(\frac{\left(\alpha^{e T} \omega^{e}\right)^{2}}{\left(\alpha^{e T} \alpha^{e}\right)^{2}}\left(I-\frac{\alpha^{e} \alpha^{e T}}{\alpha^{e T} \alpha^{e}}\right)\right) \\
& =\left(\left[\begin{array}{ccc}
\frac{1}{j_{12}} & 0 & 0 \\
0 & \frac{1}{j 22} & 0 \\
0 & 0 & \frac{1}{j 33}
\end{array}\right]-\left[\begin{array}{ccc}
0 & 0 & 0 \\
0 & \frac{\omega_{1}^{4}}{\alpha_{1}} & 0 \\
0 & 0 & \frac{\omega_{1}^{1}}{\alpha_{1}}
\end{array}\right]\right)^{-1} \\
& \left(\left[\begin{array}{ccc}
0 & 0 & 0 \\
0 & \frac{\left(\omega_{1}^{2}\right)^{2}}{\alpha_{1}^{2}} & 0 \\
0 & 0 & \frac{\left(\omega_{i}^{0}\right)^{2}}{\alpha_{1}^{2}}
\end{array}\right]\right) \\
& =\left[\begin{array}{ccc}
\frac{1}{j_{12}} & 0 & 0 \\
0 & \frac{\alpha_{1-j j_{2} \omega_{1}}}{j_{22} \alpha_{1}} & 0 \\
0 & 0 & \frac{\alpha_{1}-j_{32} \omega_{1}}{j_{33} \alpha_{1}}
\end{array}\right]^{-1}\left[\begin{array}{ccc}
0 & 0 & 0 \\
0 & \frac{\left(\omega_{1}^{2}\right)^{2}}{\alpha_{1}^{2}} & 0 \\
0 & 0 & \frac{\left.\omega_{1}^{2}\right)^{2}}{\alpha_{1}^{2}}
\end{array}\right] \\
& =\left[\begin{array}{ccc}
0 & 0 & 0 \\
0 & \frac{\left(\omega_{1}^{i}\right)^{2} j_{23}}{\alpha_{1}\left(\alpha_{1}-j_{33} \omega_{1}^{2}\right)} & 0 \\
0 & 0 & \frac{\left(\omega_{i}^{2}\right)^{2} j_{3}}{\alpha_{1}\left(\alpha_{1}-j_{33} \omega_{1}\right)}
\end{array}\right] \text {. }
\end{aligned}
$$

The skew symmetric matrix of Step $\gamma$ is

$$
S\left(\frac{\alpha^{e} \alpha^{e T}}{\alpha^{e T} \alpha^{e}} \omega^{e}\right)=\left[\begin{array}{ccc}
0 & 0 & 0 \\
0 & 0 & -\omega_{1}^{e} \\
0 & \omega_{1}^{e} & 0
\end{array}\right] .
$$


Now we compute $\mathbf{R}$ as defined in Step 8,

$$
\begin{aligned}
R & =Q_{e} J_{e} Q_{e}+\frac{\alpha^{e T} \omega^{e}}{\alpha^{e T} \alpha^{e T}}\left(I-\frac{\alpha^{e} \alpha^{e T}}{\alpha^{e T} \alpha^{e}}\right) \\
& =\left[\begin{array}{ccc}
0 & 0 & 0 \\
0 & b_{22} & 0 \\
0 & 0 & b_{33}
\end{array}\right]+\left[\begin{array}{ccc}
0 & 0 & 0 \\
0 & \frac{\omega_{1}^{i}}{\alpha_{1}} & 0 \\
0 & 0 & \frac{\omega_{i}^{i}}{\alpha_{1}}
\end{array}\right] \\
& =\left[\begin{array}{ccc}
0 & 0 & 0 \\
0 & \gamma_{22} & 0 \\
0 & 0 & \gamma_{33}
\end{array}\right]
\end{aligned}
$$

where we have

$$
\gamma_{22}=\frac{\omega_{1}^{e}}{\alpha_{1}-j_{22} \omega_{1}^{e}} \text { and } \gamma_{33}=\frac{\omega_{1}^{e}}{\alpha_{1}-j_{33} \omega_{1}^{e}} .
$$

Note that these are not the same as the $\gamma_{1}$, and $\gamma_{2}$ terms which appear in [2].
We can now compute

$$
\begin{aligned}
\mathbf{S}^{T}\left(\mathbf{r}^{e}\right) \mathbf{R S}\left(\mathbf{r}^{e}\right) & =\left[\begin{array}{ccc}
\gamma_{33} \Gamma_{2}^{e 2} & 0 & 0 \\
0 & 0 & 0 \\
0 & 0 & 0
\end{array}\right] \\
\mathbf{S}^{T}\left(\mathbf{r}^{e}\right) \mathbf{R S}\left(\mathrm{m}^{e}\right) & =0 \\
\mathbf{S}^{T}\left(\mathbf{m}^{e}\right) \mathbf{R S}\left(\mathrm{m}^{e}\right) & =\left[\begin{array}{ccc}
\gamma_{22} \mathbf{m}_{3}^{e^{2}} & 0 & 0 \\
0 & 0 & 0 \\
0 & 0 & 0
\end{array}\right] .
\end{aligned}
$$

From this we can compute the matrix $A^{T}(s) A(s)$ in (57), note that it has only Hence, $A^{T}(s) A(s)$ is a diagonal matrix and the and fourth diagonal elements. elements. As in the previous example we will nonzero eigenvalues are these two in Remark $S$.

We can construct the $D^{\prime}$ matrix in (44)

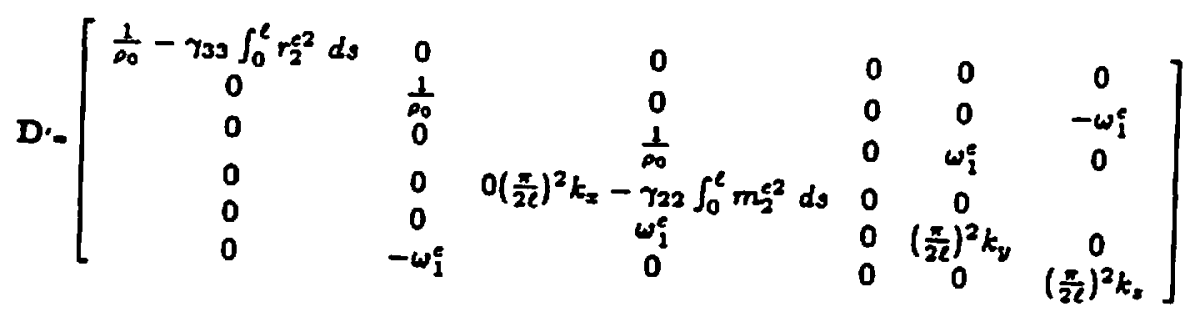


To assure that the $D^{\prime}$ matrix is positive definite we require

$$
\begin{aligned}
\frac{1}{\gamma_{33}} & >\rho_{0} \int_{0}^{\ell} r_{2}^{e 2} d s \\
\left(\frac{\pi}{2 \ell}\right)^{2} \frac{k_{x}}{\gamma_{22}} & >\int_{0}^{\ell} m_{3}^{e 2} d s
\end{aligned}
$$

and

$$
\begin{aligned}
& \frac{k_{z}}{\rho_{0}}\left(\frac{\pi}{2 l}\right)^{2}>\left(\omega_{2}^{c}\right)^{2} \\
& \frac{k_{y}}{\rho_{0}}\left(\frac{\pi}{2 l}\right)^{2}>\left(\omega_{2}^{c}\right)^{2}
\end{aligned}
$$

These conditions are exactly those of (5.14) in Krishnaprasad and Marsden and they assure stability about the equilibrium which satisfies (75)-(76).

Finally a remark about the difference between [2] and our development. If we integrate the matrix we call $A^{T}(s) A(s)$ then the elements of the integrated matrix would correspond to $\gamma_{2}$, and $\gamma_{1}$ in the paper of Krishnaprasad and Marsden. This suggests modifying the procedure in the previous section to look at the eigenvalues of the integral matrix rather than integrating the eigenvalues.

\section{Acknowledgements}

We would like to thank Prof. Juan Simo for helpful remarks.

\section{References}

[1] J.N. Franklin, Matrix Theory, Prentice-Hall, Englewood Cliff, NJ, 1968.

[2] P.S. Krishnaprasad, and J.E. Marsden, Hamiltonian Structures and Stability for Rigid Bodies with Flexible Attachments, Arch. Rat. Mech. Anal., vol.98, no.1, pp.71-93, 1987.

[3] T. Posbergh, Proposal for Ph.D. thesis, January 1986.

[4] T. Kailath, Linear Systems Theory, Prentice-Hall, Englewood Clifs, NJ, 1980.

University of Maryland

University of Maryland

University of California, Berkeley 
Copying and reprinting. Individual readers of this publication, and nonprofit libraries acting for them, are permitted to make fair use of the material, such as to copy an article for use in teaching or research. Permission is granted to quote brief passages from this publication in reviews provided the customary acknowledgement of the source is given.

Republication, systematic copying, or multiple reproduction of any material in this publication (including abstracts) is permitted only under license from the American Mathematical Society. Requests for such permission should be addressed to the Executive Director, American Mathematical Society, P.O. Box 6248, Providence, Rhode Island 02940.

The appearance of the code on the first page of an article in this volume indicates the copyright owner's consent for copying beyond that permitted by Sections 107 or 108 of the U. S. Copyright Law, provided that the fee of $\$ 1.00$ plus $\$ .25$ per page for each copy be paid directly to Copyright Clearance Center, Inc., 21 Congress Street, Salem, Massachusetts 01970. This consent does not extend to other kinds of copying, such as copying for general distribution, for advertising or promotion purposes, for creating new collective works or for resale. 